\title{
Modellierung von Bodentypen und Bodeneigenschaften im Oberengadin (Schweiz) mit Hilfe eines Geographischen Informationssystems (GIS)
}

\author{
Markus Egli, Zürich, Michael Margreth, Zürich, Urs \\ Vökt, Münchenbuchsee, Peter Fitze, Zürich, Gian- \\ franco Tognina, Chur, Felix Keller, Samedan
}

\section{Einführung}

Bodenkarten liefern wichtige Grundlagendaten für die Planung und den Vollzug der Umwelt-, Landwirtschafts- und Raumplanungsgesetzgebung (BRUNNER et al. 1997). Eine detailliertere Datengrundlage von Bodeneigenschaften in räumlicher Hinsicht ist im alpinen Raum häufig nicht vorhanden. Um einen ersten räumlichen Eindruck zu erhalten, muss auf die Bodeneignungskarte der Schweiz, welche im Massstab 1:200'000 angefertigt worden ist, zurückgegriffen werden (Frei et al. 1980). Der Nutzen und die Aussagekraft dieser Karte für die Praxis und den Vollzug ist aber nicht zuletzt bedingt durch die hohe Massstabszahl und die speziell landwirtschaftliche Ausrichtung eingeschränkt. Zudem sind die Polygone ausschliesslich als Komplexe, welche mehrere Ausprägungen pro Merkmal umfassen, dargestellt.

Ein generelles Problem stellt die hohe Variabilität der Geländeform in den Alpen (auf sehr kleiner Distanz finden sich z.B. enge Täler, Kuppen, flachere Hangstücke) dar, die entsprechende Auswirkungen auf die Bodeneigenschaften haben. Durch die ungünstige Topographie ist eine Bodenkartierung im Feld zudem mit hohen Kosten verbunden. Da für Bodenkartierungen im alpinen Raum auch in nächster Zukunft kaum die nötigen finanziellen Hilfsmittel bereit gestellt werden können, von der Gesetzgebung aber für den Vollzug genau solche Grundlagendaten notwendig sind, sind alternative Methoden bei der Datenerhebung gefragt (ToGNINA 2004). RAHMANN et al. (1997) zeigen, dass eine Bodenkartierung mittels GIS für weite und naturbelassene Gebiete in den Rocky Mountains möglich war. Diese Arbeiten beruhen auf einer Überlagerung der Geologie, Geländeeigenschaften und Vegetation zur Bestimmung von Bodentypen. Eine prozess-orientierte Modellierung mittels GIS für das Gebiet Geisenfeld (Bayern) unternahm Huber (1994). Bodenkundlich relevante Daten können auch über Fernerkundung (vgl. GauthiER \& TABBAGH 1994) abgeleitet werden. Sofern genügend Punktdaten vorhanden sind, lassen sich mittels geostatistischen Methoden Bodentypen und -eigenschaften räumlich vorhersagen (z.B. ZhU et al. 2001; AhN et al. 1999; LaGacherie \& VolTz 2000).
Das Amt für Natur und Umwelt des Kantons Graubünden stellte sich die Frage, inwieweit mit bestehenden Raumdaten, welche in digitaler Form für das Oberengadin (Abb. 1) vorliegen, ein höherer Detaillierungsgrad erzielt werden kann. Der gewünschte «Zielmassstab» sollte in einem Bereich von ca. 1:50’000 liegen. Aufgrund der bestehenden Grundlagendaten fiel eine geostatistische Modellierung von vornherein aus. Es stellte sich die Aufgabe, mit einer primär prozessorientierten Modellierung Bodentypen und -eigenschaften im hochalpinen Raum in einer für die Praxis relevanten Genauigkeit abzubilden.

\section{Methoden und Vorgehen}

\subsection{Generelles Vorgehen}

Die Bodenhypothesenkarte Oberengadin stellt eine Mischform aus Modellierung und Luftbildkartierung dar. Im Gebirgsgelände, wo das Relief innerhalb kurzer Distanz variieren kann, wurden die Bodeneigenschaften mit einem GIS-gestützten Modell abgeleitet. Ausgangspunkt für die Berechnung der Bodeneigenschaften war die Bodeneignungskarte (FreI et al. 1980). Der Miteinbezug bestehender GIS-Datensätze, Reliefanalysen und die Auswertung von Satelliten-gestützten Daten bezweckte eine Verfeinerung der Einheiten der Bodeneignungskarte 1:200'000. Die resultierende Hypothesenkarte wurde mit gezielten Stichprobenkontrollen im Feld (Abschreiten des Geländes mit kurzen Profilaufnahmen) verglichen und angepasst. Als Grundlage für die Modellierung dienten zudem 72 Bodenprofildaten aus eigenen Untersuchungen sowie aus der Literatur (MUeller 1983; Walthert et al. 2003). Die Bodeneigenschaften der Talebene wurden anhand einer Luftbildkartierung erfasst und in die GIS-gestützte Modellierung integriert. Das digitale Höhenmodell (DGM) lieferte in der Ebene zu wenig Reliefinformationen. Die Modellierung mittels GIS umfasste eine Vorhersage von Bodentypen und deren Eigenschaften. Das generelle Vorgehen zeigt Abb. 2.

\subsection{Legende der Bodenkartierung}

Die Nomenklatur der Bodeneinheiten und -eigenschaften richtet sich nach den Vorgaben von BRUNNER et al. (1997) (FAL-Nomenklatur). Reine Bodeneinheiten enthalten einen vierstelligen Code (z.B. cB1s). Dieser Code beschreibt

- den Wasserhaushalt, die pflanzennutzbare Gründigkeit (c:senkrecht durchwaschen, normal durchlässig, mässig tiefgründig), 


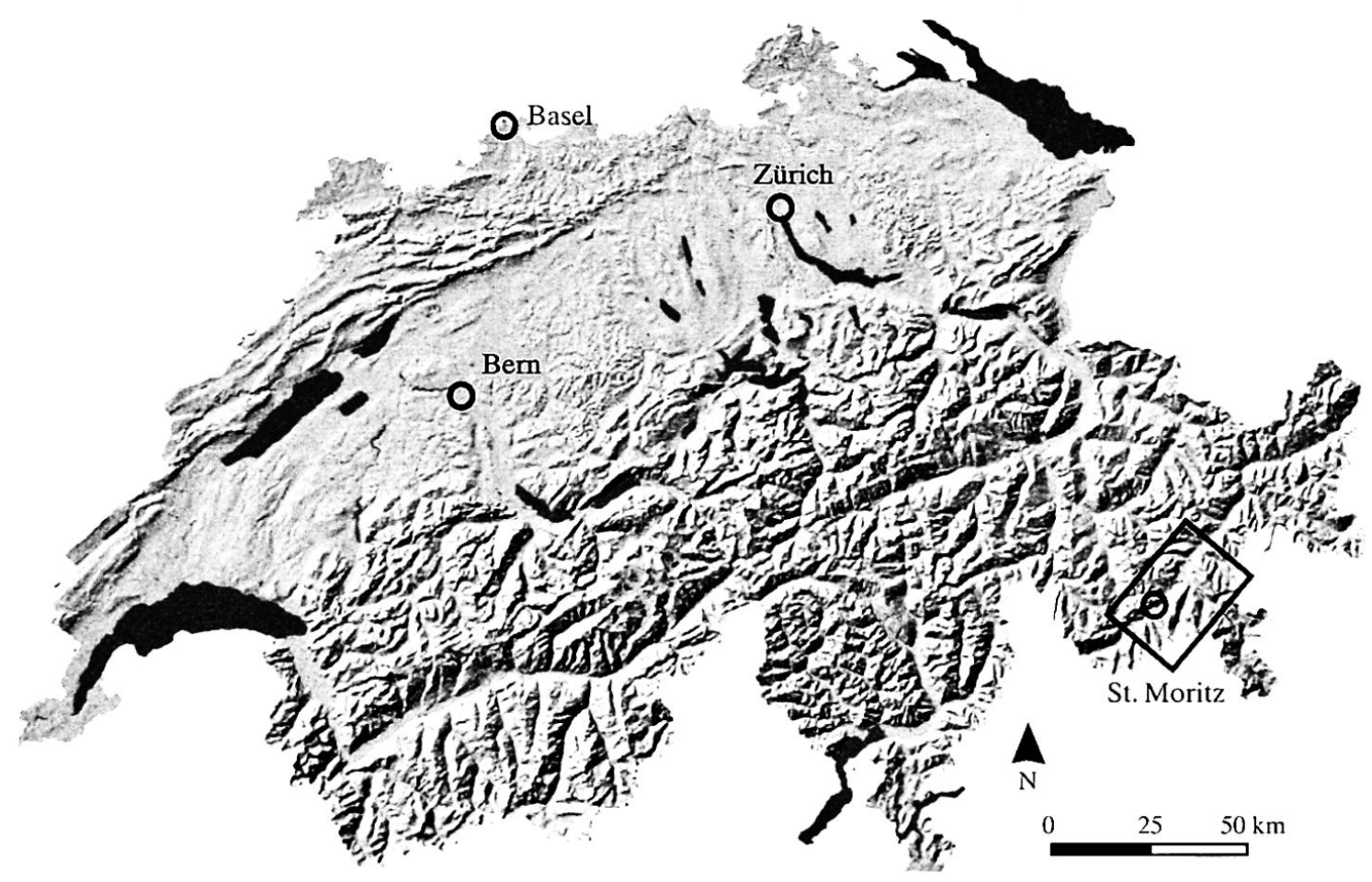

Abb. 1: Lage des Untersuchungsgebietes (Oberengadin, Schweiz)

Location of the investigation site (Upper Engadine, Switzerland)

Localisation du site d'investigation (Haut-Engadine, Suisse)

- den Bodentyp (B: Braunerde),

- die laufende Nummer (z.B. für Ausgangsmaterial, Untertyp, Skelettgehalt),

- die Geländeform (s: gleichmässig geneigt, 35-50\%).

Bodenkarten der Deutschschweiz enthalten normalerweise nebst Polygonen mit eindeutigen Zuweisungen auch solche mit mehrfacher Zuweisung (Komplexe). Komplexe Polygon-Zuweisungen sind aus datenbanktechnischer Hinsicht sowie für Modellierungen problematisch und müssen künftig weitestgehend vermieden werden (SCHÄRLI 2000).

\subsection{Modellierung \\ 2.3.1 Geländeformen}

Die Topographie bestimmt zu einem entscheidenden Masse die Bodeneigenschaften. Die Oberflächenformen wurden auf der Grundlage des DGM mit dem Modul GRID (ARC/INFO) und dort mit der Funktion CURVATURE (DENoth 1997) bestimmt. Aus den Höhenangaben des DGM kann für jede Zelle relativ zu ihren acht Nachbarzellen ein Krümmungskoeffizient errechnet werden und zwar in Richtung des grössten Gefälles wie auch parallel zu den Höhenlinien. Die Hanggeometrie wurde anschliessend mit den Hangneigungsklassen gemäss FAL kombiniert. Eine weitere Differenzierung der Topographie erfolgte durch die Ausscheidung von Nord- und Südlagen.

\subsubsection{Bodentypen}

Die für die Modellierung verwendeten Datensätze sind in Tabelle 1 aufgeführt. In einem ersten Schritt wurden für die Polygoninhalte der Bodeneignungskarte 1:200'000 eindeutige Zuweisungen generiert. Ein einzelnes Polygon der Karte 1:200'000 enthält in Bezug auf die Bodentypen mehrere Attribut-Zuweisungen (z.B. Gleysol humic, Gleysol mollic, Cambisol gleyic, Podzol). Mittels Berechnungen über GRID (Zellengrösse $20 \mathrm{x}$ $20 \mathrm{~m}$ ) wurden diese Polygone in Teilgebiete mit Dominanz der einzelnen Bodentypen aufgeteilt. Mit Hilfe von digitalen Grundlagen (wie bspw. Geländeformen, Höhenlage, Exposition) wurde der Inhalt der Polygone (bei Mehrfachzuweisungen) in eindeutige Zuweisungen umgewandelt. Aufgrund der Generalisierung (20 x $20 \mathrm{~m}$-Raster) konnte das Mikrorelief nicht berücksichtigt werden. Da die Bodeneignungskarte oberhalb der Waldgrenze nur wenig bzw. keine Polygone ausscheidet, mussten für diesen Bereich in einem weiteren Schritt die Bodentypen separat berechnet werden (Tab. 2). In Abhängigkeit des Untergrundmaterials, der Geländeform, Geländeneigung, Höhe, Wald (bzw. Vegetation), Schneeausaperungsmuster, Geomorphologie und Exposition erfolgte die entsprechende Ausscheidung der Bodentypen. Die Luftbildkartierung der Talebene umfasst die Kategorien Bodentypen, Geländeformen und Wasserhaushalt. Die 


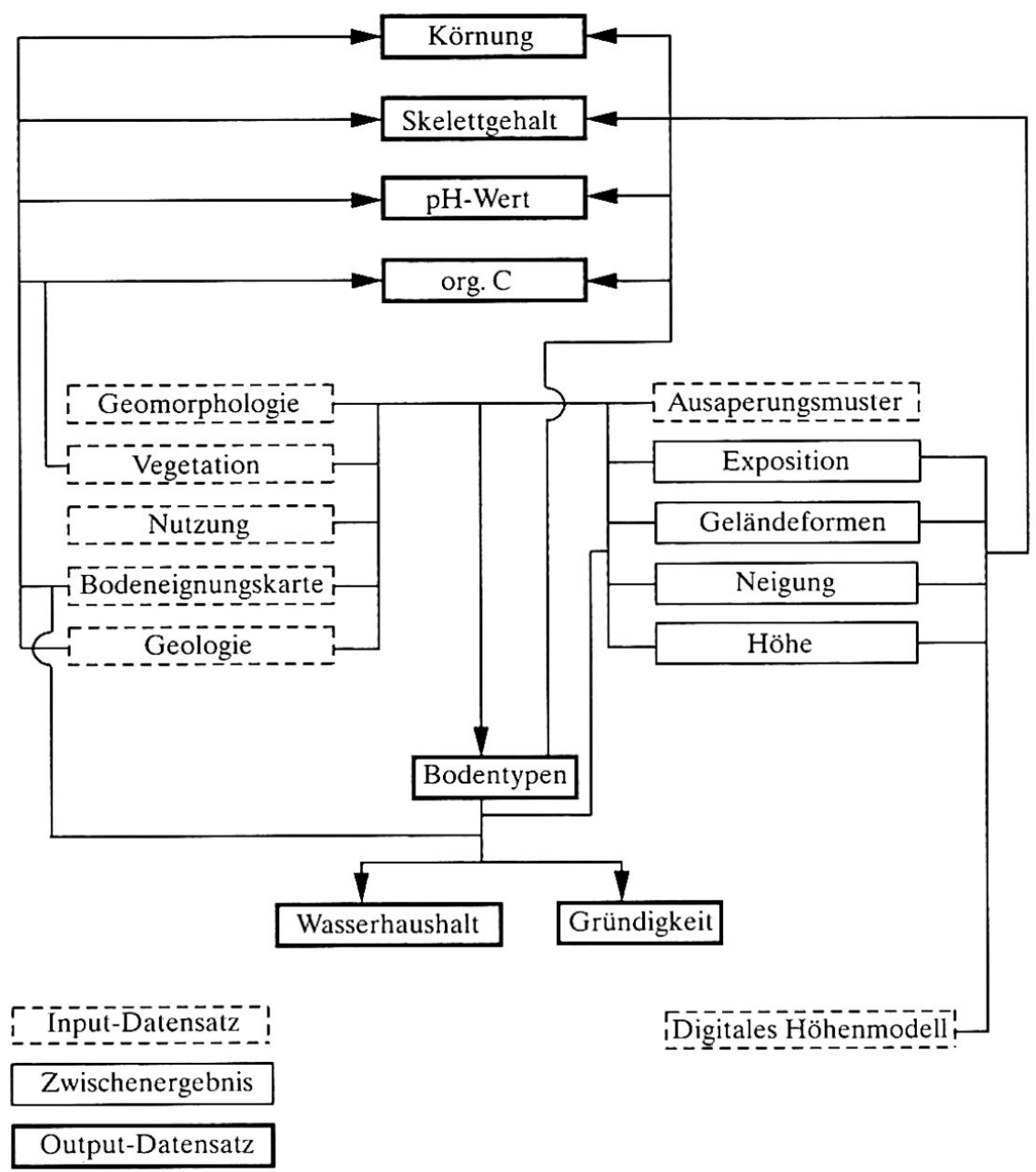

Abb. 2: Vereinfachter Datenfluss und Modellaufbau für die Berechnung der Bodentypen und -eigenschaften Simplified data flow and model concept for the calculation of soil types and properties Flux simplifié des données et concept de modèle pour le calcul des types de sol et de leurs propriétés

übrigen Bodeneigenschaften wie Gehalt an org. C, Körnung, Skelettgehalt, Gründigkeit und pH-Wert wurden berechnet (cf. Tab. 3). Die Zuweisung von Böden und Bodeneigenschaften in hanginstabilen Gebieten oder im Periglazial erfolgte über die geomorphologische Karte. Der Modellierung der chemisch-physikalischen Eigenschaften lag die Annahme zugrunde, dass Bodentypen, bedingt durch ihre Evolution, jene in ihrer Summe widerspiegeln. Unter Berücksichtigung der Höhenlage, der Geologie, der Neigung und/oder der Geländeform erfolgte eine entsprechende Feingliederung (vgl. Tab. 3 und 4). Eigenschaften, wie z.B. der org. Kohlenstoffgehalt im Boden, sind eine Kombination von Bodentransferfunktionen und Bodentypen (Tab. 3). Die Verwertung der einzelnen Datensätze erfolgte nach dem entity relationshipPrinzip (vgl. Sauer 1992; KLINGl 1996), d.h. durch die Auswahl der relevanten Attribute und die Definition der jeweiligen Relationen. Die einzelnen Ausdrücke der Abfrage sind mit Booleschen Funktionen, nämlich AND, OR, NOT sowie logischen Operatoren wie «lower than», «lower equal», «equal», «not equal», «greater equal», «greater than» (KLINGL 1996) realisiert worden.

\subsubsection{Gründigkeit und Wasserhaushalt}

Die Gründigkeit und der Wasserhaushalt eines Bodens wurden über zwei verschiedene Verfahren bestimmt. A) Die Ableitung der gewünschten Eigenschaften erfolgte über eine Kombination der Bodeneignungskarte (FrEI et al.1980) mit dem DGM. B) Ausserhalb des kartierten Bereichs der Bodeneignungskarte basierte die Berechnung auf einer Überlagerung der modellierten Bodentypen mit dem Geländemodell.

\subsubsection{Organischer Kohlenstoff}

Die Menge an org. $\mathrm{C}$ im Boden kann als Funktion der Höhe dargestellt werden (Hirz 2002). Die von Hitz (2002) für das Vereinatal abgeleitete Funktion wurde 


\begin{tabular}{|ll|}
\hline Inhalt & Massstab \\
\hline Geländemodell (20 m-Auflösung) & $1: 25000$ \\
Klima/Wasserhaushalt & \\
Geländemodell (20 m-Auflösung) & $1: 25000$ \\
Fluviatiler Prozessbereich & $1: 25000$ \\
Glaziofluviatiler Prozessbereich & $1: 25000$ \\
Periglazialer Prozessbereich & $1: 25000$ \\
Glazialer Prozessbereich & $1: 25000$ \\
Glaziologische Karte & $1: 25000$ \\
Ausaperungsmuster (Spot) & $20 \mathrm{~m}$ \\
Geologie/Geomorphologie & $1: 200000$ \\
Geotechnische Karte & $1: 200000$ \\
Geologie in 4 Klassen (Gletscher/Seen, & $1: 200000$ \\
Silikat, Karbonat, Mischgestein) & $1: 25000$ \\
Rutschungen & $1: 25000$ \\
Lawinenzüge & $1: 25000$ \\
Organogener Prozessbereich & $1: 25000$ \\
Gravitativer Prozessbereich & $1: 25000$ \\
Murgang Anrissgebiete & $1: 25000$ \\
Murgang Ablagerungsgebiete & $1: 200000$ \\
Murgang Transportgebiete & Punktdaten \\
Bodeneignungskarte & $1: 50000$ \\
Im Feld erhobene Bodenprofildaten \\
Feldkartierung Boden im Tal & $1: 10000$ \\
Vegetation/Nutzung & $1: 10000$ \\
Flachmoorinventar & $1: 10000$ \\
Hochmoorinventar & $1: 25000$ \\
Biotopdetailkartierungen & $1: 25000$ \\
Aueninventar & $1: 25000$ \\
Alpine Auen & $1: 25000$ \\
Trockenwiesen und -weiden & \\
Wald & $1: 5000$ \\
Sonstiges & $1: 25000$ \\
Siedlungsgebiete & \\
Golfanlagen (aus Nutzungsplanung) \\
Seen & 1 Fliessgewässer \\
\hline
\end{tabular}

Tab. 1: GIS-Datengrundlage, aufgegliedert nach den bodenbildenden Faktoren

GIS data base listed according to soil forming factors

Base de donnée SIG listée d'après les facteurs de la formation du sol für das Oberengadin übernommen und an vorhandene Profil-Daten angepasst (Tab. 3).

\subsection{5 pH-Wert und Skelettgehalt}

Die Modellierung des pH-Wertes und des Skelettgehaltes wurde auf der Basis der Bodentypen, der Topographie und der Gesteinsunterlage (Tab. 4, Abb. 2) durchgeführt.

\section{Resultate und Diskussion}

Die flächenhafte Ausscheidung von Bodentypen wird im allgemeinen nach dem Catena-Prinzip (Döbel 1998; LESER 1997) durchgeführt. Die flächenhafte Aufnahme von Böden und deren Eigenschaften ist in heterogenen Gebieten, wie sie die Alpen repräsentieren, jedoch stark erschwerten Bedingungen ausgesetzt (vgl. DöBel 1998). Der Aufnahme geomorphographischer und -dynamischer Merkmale sowie im speziellen der Bestimmung von Geländeformen und -neigungen kommt in besonders stark reliefierten Hochgebirgsregionen eine grosse Bedeutung zu, weil sie in enger Beziehung zu den Böden und ihren Eigenschaften stehen (vgl. DA Rосна 1981). In Gebirgsregionen ist eine Modellierung der Geländeformen der Feldkartierung vorzuziehen. Die Geländeformen können einheitlich definiert werden und ohne grossen Aufwand für grössere Gebieten in all ihren Facetten modelliert werden (vgl. RahmanN et al. 1997; Gessler et al. 2000).

Durch die definierte Modellstruktur wird eine bedeutende Verfeinerung der Bodeneignungskarte (1:200’000) erreicht. Die neu erzeugten Raster haben eine eindeutige Zuweisung für den Bodentyp, die Geländeform, Bodeneigenschaften (org. C, pH-Wert, Skelettgehalt, Körnung der Feinerde; vgl. Abb. 3) und den Wasserhaushalt/Gründigkeit. Die Auflösung der Teilfä̈chen wurde zudem um ein Vielfaches erhöht. Ein quantitativer Vergleich der Modellergebnisse mit den erhobenen Profilen (ausserhalb der Talebene) zeigt, dass exakte Treffer (Vergleich Modell - Feld) selten realisierbar sind. Werden aber kleinere Abweichungen als tolerierbar angenommen, d.h. eine gewisse Streubreite impliziert, so haben die Vorhersagen mit dem Modell für die Bodeneigenschaften wie Gründigkeit, $\mathrm{pH}$-Wert, Skelettgehalt und Körnung eine Genauigkeit im Rahmen von rund 70-80\% (Tab. 5). Die quantitative Überprüfung des Modells erfolgte auf der Basis von Punktdaten (Bodenprofilen). Diese haben einen stark lokalen Bezug. Die Zellgrösse der Modellierung beträgt $20 \times 20 \mathrm{~m}$; die Dimensionen einer Profilgrabung ca. $2 \times 2 \mathrm{~m}$. Das Mikrorelief und die Bodenbildung können innerhalb weniger Meter variieren. Die Genauigkeit der Modellierung hängt somit stark von der räumlichen Auflösung des DGM ab. Visuelle 


\begin{tabular}{|c|c|c|}
\hline Grundlagen & Funktion, Tätigkeit & Output \\
\hline \multirow[t]{4}{*}{ Gelände } & Ableitung, Klassierung & Neigung \\
\hline & Curvature (horizontal/vertikal) & Geländeformen \\
\hline & Klassierung & Exposition \\
\hline & Überlagerung Curvature-Neigung & FAL-Formen \\
\hline Vegetation & & $\begin{array}{l}\text { Flach- und Hochmoore } \\
\text { Alpine Auen }\end{array}$ \\
\hline Geologie & $\begin{array}{l}\text { Klassierung nach Chemie } \\
\text { des Ausgangsmaterials }\end{array}$ & $\begin{array}{l}\text { silikatisch } \\
\text { silikatisch + karbonatisch } \\
\text { karbonatisch }\end{array}$ \\
\hline Geomorphologie & & $\begin{array}{l}\text { Prozessbereiche: } \\
\text { glazial } \\
\text { periglazial } \\
\text { fluviatil } \\
\text { gravitativ } \\
\text { organogen }\end{array}$ \\
\hline Glaziologie & & $\begin{array}{l}\text { Gletscher } \\
\text { Gletschervorfelder }\end{array}$ \\
\hline Schneebedeckung im Juni ${ }^{1)}$ & Klassierung, Überlagerung Gelände & Ausaperungsmuster klassiert \\
\hline Bodenprofile & $\begin{array}{l}\text { Eigene Erhebungen } \\
\text { Kompilation aus Literatur }\end{array}$ & $\begin{array}{l}\text { Bodenprofilaufnahmen } \\
\text { Bodenprofilaufnahmen }\end{array}$ \\
\hline Output der Grundlagen & Überlagerung, BooLsche Operatoren & Hypothetische Verteilung der Bodentypen \\
\hline
\end{tabular}

Tab. 2: Vorgehen zur Berechnung der Bodentypen im hochalpinen Raum (oberhalb der Waldgrenze) Procedure for the derivation of soil types in the high-alpine zone (above timberline)

Procédure destinée au calcul de la dérivation des types de sol dans la zone alpine (au-dessus de la limite forestière)

\begin{tabular}{|c|c|c|}
\hline Bodentypen & Gebiet & Funktion \\
\hline Eisenpodsol, Eisenhumuspodsol & Wald & $\begin{array}{l}y=-0.00002367 x^{2}+0.0954 x- \\
76.17249\end{array}$ \\
\hline $\begin{array}{l}\text { Saure Braunerde, Braunpodsol, } \\
\text { Braunerde-Gley, Buntgley }\end{array}$ & $\begin{array}{l}\text { Wald, geschlossenes } \\
\text { Grasland }\end{array}$ & $\begin{array}{l}y=-0.00002152(x-200)^{2}+ \\
0.07812(x-200)-52.76292^{1)}\end{array}$ \\
\hline $\begin{array}{l}\text { Regosole, Phaeozem, Braunerde, } \\
\text { Kalkbraunerde, Rendzina, Fahlgley, } \\
\text { Fluvisole }\end{array}$ & & $\begin{array}{l}y=\left(-0.00002152(x-200)^{2}+\right. \\
0.07812(x-200)-52.76292) * 0.71\end{array}$ \\
\hline $\begin{array}{l}\text { Aueböden, Fluvisole, Humus-Silikat- } \\
\text { gesteinsboden, Humus-Kalkgesteinsboden, } \\
\text { Humus-Mischgesteinsboden }\end{array}$ & & $\begin{array}{l}y=\left(-0.00002152(x-200)^{2}+\right. \\
0.07812(x-200)-52.76292) * 0.31\end{array}$ \\
\hline $\begin{array}{l}\text { Silikatgesteinsboden, Kalkgesteinsboden, } \\
\text { Mischgesteinsboden }\end{array}$ & $\begin{array}{l}\text { Schutthalden, } \\
\text { Lawinenzüge }\end{array}$ & $\begin{array}{l}y=\left(-0.00002152(x-200)^{2}+\right. \\
0.07812(x-200)-52.76292) * 0.125\end{array}$ \\
\hline
\end{tabular}

$\mathrm{x}=$ Meter ü.d.M.

$\mathrm{y}=$ Kohlenstoffgehalt im Boden in $\mathrm{kg} / \mathrm{m}^{2}$

1) Korrelationskoeffizient für das Vereinatal $=0.62$ und $p<0.001$

Tab. 3: Modellierung der org. C-Menge im Boden (modifiziert nach Hitz 2002) 


\begin{tabular}{|c|c|c|c|c|}
\hline Code & Bodentyp & Bedingungen & $\begin{array}{c}\text { pH-Wert } \\
\text { Oberboden }^{\text {1) }}\end{array}$ & $\begin{array}{c}\text { pH-Wert } \\
\text { Unterboden } / \mathrm{C}\end{array}$ \\
\hline \multirow[t]{2}{*}{ A } & Aueboden & Silikatgestein & 5 & 5 \\
\hline & & Mischgestein, Karbonatgestein & 7.5 & 7.5 \\
\hline B & Braunerde & & 5.5 & 6.5 \\
\hline $\mathrm{C}$ & Humus-Karbonatgesteinsboden & & 7 & 7.5 \\
\hline $\mathrm{D}$ & Humus-Mischgesteinsboden & & 6.5 & 7 \\
\hline $\mathrm{E}$ & Saure Braunerde & & 4 & 4.5 \\
\hline \multirow[t]{2}{*}{$\mathrm{F}$} & Fluvisol & Silikatgestein & 5 & 5 \\
\hline & & Mischgestein, Karbonatgestein & 7.5 & 7.5 \\
\hline G & Fahlgley & & 5 & 6 \\
\hline $\mathrm{H}$ & Humuspodsol & & 3.5 & 4 \\
\hline I & Pseudogley & nicht vorkommend & & \\
\hline $\mathrm{J}$ & Karbonatgesteinsboden & & 7 & 7.5 \\
\hline $\mathrm{K}$ & Kalkbraunerde & & 6 & 7 \\
\hline $\mathrm{L}$ & Silikatgesteinsboden & & 5 & 5 \\
\hline \multirow[t]{2}{*}{ M } & Moor & Silikatgestein & 3.5 & \\
\hline & & Mischgestein, Karbonatgestein & 7.5 & \\
\hline \multirow[t]{2}{*}{$\mathrm{N}$} & Halbmoor & Silikatgestein & 3.5 & \\
\hline & & Mischgestein, Karbonatgestein & 7.5 & \\
\hline $\mathrm{O}$ & Regosol & & 6.5 & 7 \\
\hline$P$ & Eisenpodsol & & 3.3 & 4 \\
\hline Q & Braunpodsol & & 4 & 4 \\
\hline $\mathrm{R}$ & Rendzina & & 6.5 & 7.5 \\
\hline $\mathrm{S}$ & Humus-Silikatgesteinsboden & & 4.5 & 5 \\
\hline $\mathrm{T}$ & Parabraunerde & & 5.5 & 6.5 \\
\hline $\mathrm{U}$ & Mischgesteinsboden & & 6.5 & 7 \\
\hline V & Braunerde-Gley & & 4.5 & 5.5 \\
\hline W & Buntgley & & 4.5 & 5.5 \\
\hline $\mathrm{X}$ & Auffüllung & nicht klassiert & & \\
\hline $\mathrm{Y}$ & Braunerde-Pseudogley & nicht vorkommend & & \\
\hline $\mathrm{Z}$ & Phaeozem & & 5 & 6 \\
\hline \multicolumn{5}{|c|}{ Bodenfreie Gebiete } \\
\hline & Silikatgestein & & 5 & \\
\hline & Mischgestein & & 7.5 & \\
\hline & Karbonatgestein & & 7.5 & \\
\hline
\end{tabular}

Tab. 4: Berechnung des $\mathrm{pH}$-Wertes für den Ober- und Unterboden/Ausgangsmaterial

Determination of the $\mathrm{pH}$-values of topsoil and subsoils/parent material

Détermination de la valeur du $\mathrm{pH}$ de la couche supérieure et du sous-sol altéré/roche-mère

Vergleiche im Feld zeigten, dass die flächenbezogene Genauigkeit wahrscheinlich besser ist. Ein wirklich quantitativer, flächenhafter Vergleich zwischen modellierten und kartierten Bodeneigenschaften (vgl. EGLI et al. 2004) war jedoch nicht möglich. Ein effektiver Vergleich zwischen Modell und Wirklichkeit ist somit nur bedingt realisierbar. Eine ähnliche Arbeit (EGL1 et al. 2004), jedoch in gemässigten Klimazonen, belegt, dass mittels GIS-Modellierung zum Teil bessere Übereinstimmungen mit Karten einer kleinen Massstabszahl (1:5’000) erzielt werden als mit Karten grösserer Massstabszahlen (1:25’000). Die Modellierung von Bodeneigenschaften und Bodentypen unter Miteinbezug des DGM ist v.a. für das Gebirgsgelände mit scharfen Konturen geeignet. Im Flachland, wo die Geländeunterschiede wesentlich geringer sind, ist die Luftbildkartierung effizienter. Die Ableitung von Geländeformen basierend auf geringen Reliefunterschieden ist über die Luftbildkartierung genauer. Der $20 \times 20 \mathrm{~m}$-Raster ist dazu weniger geeignet. Eine höhere Auflösung des Geländemodells könnte diesem Problem jedoch Abhilfe schaffen. 


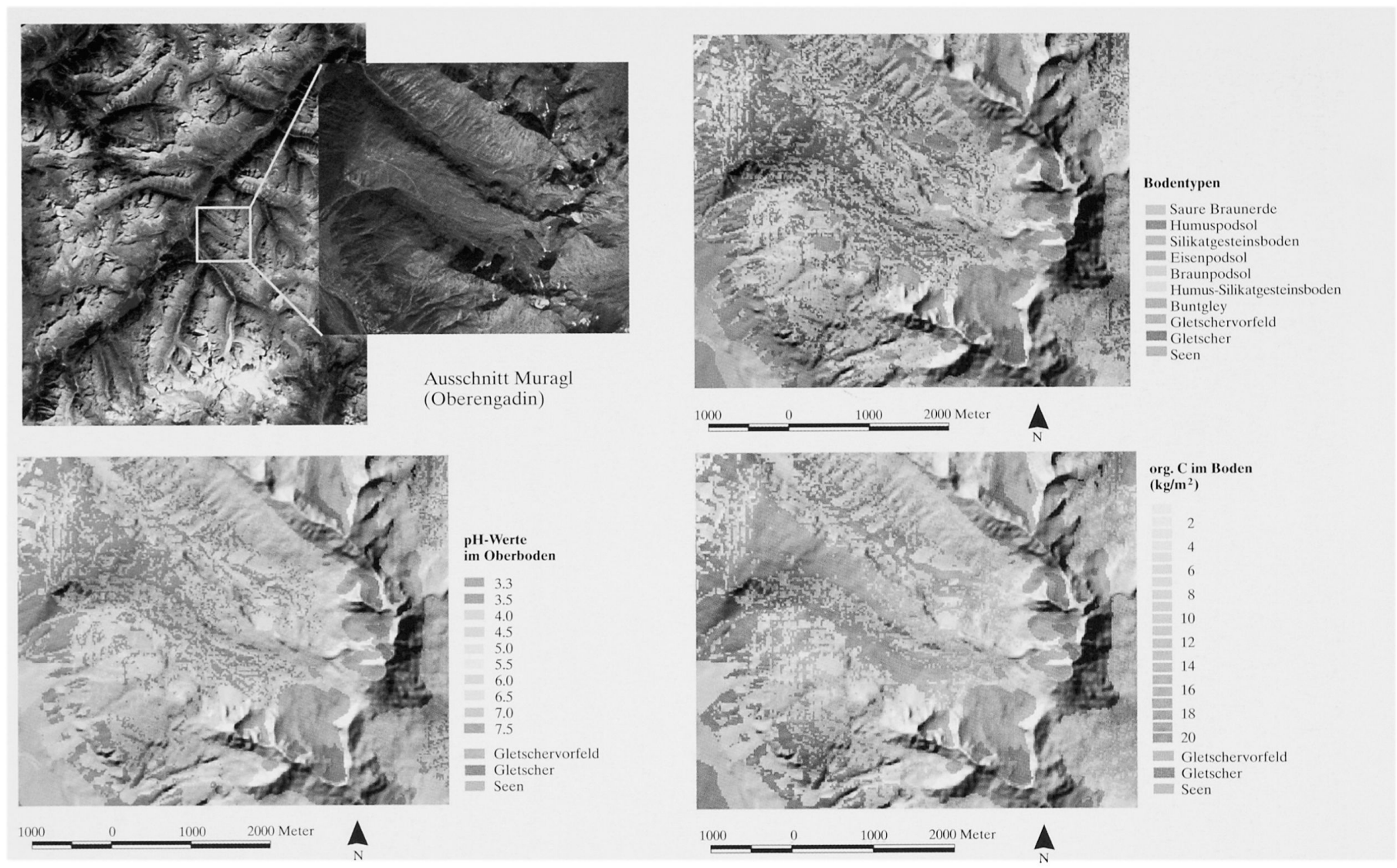

Abb. 3: Modellierte Bodentypen, $\mathrm{pH}$-Werte im Oberboden und org. C-Gehalt $\left(\mathrm{kg} / \mathrm{m}^{2}\right)$ im Ausschnitt Muragl (Oberengadin) Modelled soil types, $\mathrm{pH}$-values in the topsoil and org. C content $\left(\mathrm{kg} / \mathrm{m}^{2}\right)$ in the region of Muragl (Upper Engadine)

Types de sol modelés, valeurs du pH dans les couches supérieures du sol et taux du C organique dans le secteur de Muragl (Haut-Engadine)

Quelle: Satellitenbild (Landsat 7) vom 13.9.1999; Satellitendaten: (C) ESA/Eurimage/swisstopo, NPOC; Bildverarbeitung: F. PAUL 


\begin{tabular}{|c|c|c|c|}
\hline $\begin{array}{l}\text { Modellierte } \\
\text { Grösse }\end{array}$ & Items & Spezifikationen & $\begin{array}{l}\text { Übereinstimmung } \\
\text { Modell - Feld in \% }\end{array}$ \\
\hline Bodentypen & & $\begin{array}{l}\text { allgemein } \\
\text { Podsole }\end{array}$ & $\begin{array}{l}65 \\
75 \\
\end{array}$ \\
\hline \multirow[t]{18}{*}{$\begin{array}{l}\text { Bodeneigen- } \\
\text { schaften }\end{array}$} & Gründigkeit & $\begin{array}{l}\text { exaktes Zutreffen } \\
\text { Abweichung } 1 \text { Gründigkeitsklasse } \\
\text { Summe }\end{array}$ & $\begin{array}{l}28 \\
50 \\
78\end{array}$ \\
\hline & $\mathrm{pH}$ (Oberboden) & $\begin{array}{l}\text { exaktes Zutreffen } \\
\text { Abweichung }<0.2 \mathrm{pH} \text {-Einheit }\end{array}$ & $\begin{array}{r}6 \\
28\end{array}$ \\
\hline & & Abweichung $\geq 0.2$ und $<0.5 \mathrm{pH}$-Einheit & 28 \\
\hline & & Summe & 62 \\
\hline & $\mathrm{pH}$ (Unterboden) & exaktes Zutreffen & 6 \\
\hline & & Abweichung $<0.2 \mathrm{pH}$-Einheit & 17 \\
\hline & & Abweichung $\geq 0.2$ und $<0.5 \mathrm{pH}$-Einheit & 44 \\
\hline & & Summe & 67 \\
\hline & Skelettgehalt (Oberboden) & $\begin{array}{l}\text { exaktes Zutreffen } \\
\text { Abweichung }<10 \% \\
\text { Abweichung } \geq 10 \text { und }<20 \%\end{array}$ & $\begin{array}{r}12 \\
71 \\
6\end{array}$ \\
\hline & & Summe & 89 \\
\hline & Skelettgehalt (Unterboden) & $\begin{array}{l}\text { exaktes Zutreffen } \\
\text { Abweichung }<10 \% \\
\text { Abweichung } \geq 10 \text { und }<20 \%\end{array}$ & $\begin{array}{r}0 \\
39 \\
39\end{array}$ \\
\hline & & Summe & 78 \\
\hline & Körnung (Oberboden) & exaktes Zutreffen & 25 \\
\hline & & Abweichung 1 Körnungsklasse & 44 \\
\hline & & Summe & 69 \\
\hline & Körnung (Unterboden) & exaktes Zutreffen & 47 \\
\hline & & Abweichung 1 Körnungsklasse & 33 \\
\hline & & Summe & 80 \\
\hline
\end{tabular}

1) Summe = akzeptables Ergebnis, bestehend aus der Summe der Eintretenswahrscheinlichkeiten

Tab. 5: Übereinstimmung zwischen Modellergebnissen und Feldaufnahmen (Bodenprofile, $\mathrm{n}=52$ ) A quantitative comparison between model results and field observations (soil profiles, $n=52$ ) Concordance des résultats obtenus par la modélisation et les observations sur le terrain (profils des sols, $n=$ 52)

\section{Schlussfolgerungen}

Die Verarbeitung GIS-basierter Grundlagen führte $\mathrm{zu}$ einer wesentlichen Verbesserung der Bodeneignungskarte 1:200’000. Die Verbesserungsmöglichkeiten hängen stark vom Datensatz und dem jeweiligen Erfassungsmassstab der Daten ab. Im gezeigten Fall variierte die Massstabszahl der Grundlagedaten im Bereich von $10^{\prime} 000$ bis $200^{\prime} 000$. Somit ist es durchaus möglich, dass gewisse Attribute einer Bodenkartierung genauer erfasst werden können als andere. Die Modellierung der Bodentypen, des Wasserhaushaltes und der pflanzennutzbaren Gründigkeit erfolgte zufriedenstellend (Tab. 5). Die Modellierung weiterer Bodeneigenschaften wurde von Bodentransferfunktionen (org. C), von den Bodentypen und/oder den Geländeeigenschaften abhängig gemacht. Überprüfungen mit Feldbegehungen und bestehenden Profildaten zeigen eine gute Übereinstimmung. Der Massstab der Karte wurde vorsichtig auf 1:50'000 geschätzt, dürfte aber wahrscheinlich besser sein. Die GIS-gestützte Modellierung von Bodeneigenschaften und Luftbildkartierung lassen sich gut kombinieren. Die beiden Methoden können gemäss ihren Stärken gezielt und flexibel eingesetzt werden und lassen sich somit gut ergänzen. Die modellierte Bodenkarte ist 
eine Hypothesenkarte. Eine Verbesserung des Modells könnte über zusätzliche Bodentransferfunktionen oder Boden-Klimasequenzen erzielt werden. Eine weitere Möglichkeit wäre die Kartierung repräsentativer, jedoch flächenmässig kleiner Gebiete und deren Extrapolation auf grössere Einheiten beispielsweise mittels probabilistischen Funktionen (LAgacherIE \& VolTZ 2000).

Im Zusammenhang mit Bauvorhaben und raumplanerischen Fragen können mit dieser Karte bereits recht detaillierte Angaben gemacht werden. Die Hypothesenkarte kann eine Feldkartierung aber nicht gänzlich ersetzen. Zur Klärung rechtlicher und planerischer Fragen im Hinblick auf spezifische und lokale Probleme sind Detailkartierungen im Feld nach wie vor unabdingbar.

\section{Dank}

Diese Arbeit wurde durch finanzielle Beiträge des Kantons Graubünden, des Kreises Oberengadin, der Academia Engiadina und des Schweizerischen Nationalfonds (NFP48) ermöglicht.

\section{Literatur}

Ahn, C.W., Baumgardner, M.F. \& L.L. Biehl (1999): Delineation of soil variability using geostatistics and fuzzy clustering analyses of hyperspectral data. - In: Soil Science Society of America Journal 63: 142-150.

BrunNer, J.,JäGGli, F., Nievergelt, J. \& K. Peyer (1997): Kartieren und Beurteilen von Landwirtschaftsböden.$=$ Schriftenreihe der FAL (Eidgenössische Forschungsanstalt für Agrarökologie und Landbau) 24, ZürichReckenholz.

Da Rocha, H.O. (1981): Die Böden und geomorphologischen Einheiten der Region von Curitiba (Paraná-Brasilien). - = Freiburger bodenkundliche Abhandlungen 10, Freiburg im Breisgau: Institut für Bodenkunde und Waldernährungslehre der AlbertLudwigs-Universität.

DENOTh, F. (1997): Oberflächenabfluss und Phosphorverlust auf landwirtschaftlich genutzten Flächen. = Unveröffentlichte Diplomarbeit, Geographisches Institut, Universität Zürich.

DöBELI, C. (1998): Bodenkartierung im Untersuchungsgebiet der Gemmi (Walliser Alpen) mit Hilfe geomorphologischer Methoden. - In: Vischer, D. (Hrsg.): Beiträge aus der Gebirgs-Geomorphologie, Mitteilungen der VAW 158, Zürich: 42-54.

Egli, M., Häfliger, S. \& M. Achermann (2004): Modellierung von Bodenkarten: Ansätze zur Verbesserung der bestehenden Grundlagen. - In: Bulletin BGS 27: 45-48.

Frei, E., Vökt, U., Flückiger, R., Brunner, H. \& F. Schai (1980): Bodeneignungskarte der Schweiz,
Massstab 1:200'000. - = Grundlagen für die Raumplanung, Bundesämter für Raumplanung, Landwirtschaft und Forstwesen, EDMZ Bern.

GAuthier, F. \& A. TABbagh (1994): The use of airborne thermal remote sensing for soil mapping: a case study in the Limousin region (France). - In: International Journal of Remote Sensing 15: 1981-1989.

Gessler, P.E., Chadwick, O.A., Chamran, F., Althouse, L. \& K. Holmes (2000): Modeling soil-landscape and ecosystem properties using terrain attributes. - In: Soil Science Society of America Journal 64: 2046-2056.

Hirz, C. (2002): Inventur und Dynamik der organischen Substanz in Böden der alpinen Stufe. Untersuchungen im Vereina-Tal, GR, Schweiz. - = Schriftenreihe Physische Geographie 42, Geographisches Institut der Universität Zürich.

Huber, M. (1994): The digital geo-ecological map. Concepts, GIS-methods and case studies. - In: Basler Beiträge zur Physiogeographie, Physiogeographica 20: 1-144.

KLINGL, T. (1996): GIS-gestützte Generierung synthethischer Bodenkarten und landschaftsökologische Bewertung der Risiken von Bodenwasser- und Bodenverlusten. $-=$ Geographica Bernensia G50, Bern.

Lagacherie, P. \& M. Voltz (2000): Predicting soil properties over a region using sample information from a mapped reference area and digital elevation data: A conditional probability approach. - In: Geoderma 97: 187-208.

LeSER, H. (1997): Landschaftsökologie: Ansatz, Modelle, Methodik, Anwendung. - 4. Auflage, Stuttgart: Eugen Ulmer.

Mueller, M. (1983): Bodenbildung auf Silikatunterlage in der alpinen Stufe des Oberengadins. - = Dissertation Naturwissenschaften, ETH Zürich.

Rahmann, S., Munn, L.C., Vance, G.F. \& C. Arneson (1997): Wyoming rocky mountain forest soils: mapping using ARC/INFO Geographic Information System. - In: Soil Science Society of America Journal 61: 1730-1737.

SAUER, H. (1992): Relationale Datenbanken. - Bonn: Addison-Wesley.

SCHÄrLI, J. (2000): Einheitliche Bodenlegenden für Bodenkarten des Kantons LU als Basis für ein Geografisches Informationssystem (GIS). - = Unveröffentlichte Diplomarbeit, Hochschule Technik + Architektur, Luzern.

Tognina, G. (2004): Hilfsmittel Bodenkarte: Methodik, Realisierbarkeit, Anwendungspotential am Beispiel eines Gebirgskantons. - In: Bulletin BGS 27: 49-52.

Walthert, L., Blaser, P., Lüscher, P., Luster, J. \& S. ZimmermanN (2003): Langfristige Waldökosystem-Forschung LWF in der Schweiz, Kernprojekt Bodenmatrix: Ergebnisse der ersten Erhebung 1994-1999. - http://ecollection.ethbib.ethz.ch/show?type=bericht $\& n r=276$, April 2003. 
Zhu, A.X, Hudson, B., Burt, J., Lubich, K. \& D. Somonson (2001): Soil mapping using GIS, expert knowledge and fuzzy logic. - In: Soil Science Society of America Journal 65: 1463-1472.

\section{Zusammenfassung: Modellierung von Bodentypen und Bodeneigenschaften im Oberengadin (Schweiz) mit Hilfe eines Geographischen Informationssystems (GIS)}

Detaillierte Bodenkarten im schweizerischen Alpenraum existieren praktisch nicht. Die einzige Grundlage ist die Bodeneignungskarte im Massstab 1:200'000. Mit der GIS-Datenbasis des Kantons Graubünden wurde versucht, über eine räumliche Analyse einen Teil der Bodeninformationen nach der FAL-Nomenklatur zu modellieren. Die modellierten Grössen umfassen Bodentyp, Gründigkeit, Wasserhaushalt, Geländeform, pH-Wert, org. C-Gehalt, Skelettgehalt und die Körnung. Die Berechnungen basieren zu einem Teil auf der Bodentypenmodellierung, von welcher weitere Eigenschaften abgeleitet werden konnten. Die Modellierung stützt sich auf das prozess-orientierte entity relationship-Prinzip sowie auf Pedo-Transfer-Funktionen. Da die Rasterweite des digitalen Höhenmodells von 20 Metern für die Modellierung der Bodeneigenschaften der Talebene nicht befriedigen konnte, wurden die Bodeneigenschaften in der Talebene mit einer Luftbildkartierung erfasst. Die erzielten Resultate stimmen zuversichtlich (die Übereinstimmung Feld - Modell liegt bei ca. 70\%) und können in einem auch für die Praxis relevanten Massstab von ca. 1:50’000 dargestellt werden.

\section{Abstract: Modelling of soil properties in the Upper Engadine (Switzerland) using a geographic informa- tion system (GIS)}

Detailed soil maps of the Swiss Alps are rare. The only existing soil database for such areas is a map with a scale of 1:200'000. Using the GIS data base of the Canton of Grisons, a spatial analysis was made with the aim of putting together at least part of the information needed for a soil map in the Alpine area Upper Engadine. Soil classification followed FAL terminology. Factors included in the model were soil type, soil depth relevant for plant growth, soil hydrology, terrain form, $\mathrm{pH}$-value, organic $\mathrm{C}$ content, soil skeleton and granulometry. To a large extent, the modelled soil types were used for the calculation and derivation of further soil properties. The modelling is based on the principle of process-oriented entity relationships and on the use of pedo transfer functions. As the grid width of the DEM $(20 \mathrm{~m})$ was unsatisfactory for modelling the soil properties of the main valley floor, aerial photographs were used to derive the latter. The obtained results correspond well with field measurements (agreement around 70\% between modelled and measured properties) and reflect a useful scale (about 1:50’000) for practical implementation.
Résumé: Modélisation des types de sols et des propriétés du sol dans le Haut-Engadine (Suisse) au moyen d'un système d'information géographique (SIG)

Dans la plupart des cas, il n'existe pratiquement pas de cartes détaillées de sols pour les régions alpines suisses. La seule base de données pour ces régions est la carte d'aptitude des sols au 1:200'000. Nous avons essayé d'effectuer, à l'aide de la base de données SIG du Canton des Grisons, une analyse spatiale pour modéliser une partie des informations sur le sol, selon la nomenclature FAL. Les grandeurs modélisées sont les suivantes: type de sol, profondeur du sol, hydrologie du sol, forme du terrain, valeur $\mathrm{du} \mathrm{pH}$, contenu de la matière organique $\mathrm{C}$, proportion de la charpente et granulométrie. Les calculs se fondent en partie sur la modélisation des types de sols, une modélisation dont on peut déduire d'autres propriétés. La modélisation se base sur le principe de l'«entity relationship» et sur des fonctions «pedo-transfer». Comme la résolution du modèle d'élévation numérique du terrain $(20 \mathrm{~m})$ pour la vallée n'était pas suffisante. Pour cette raison, la cartographie propriétés des sols au fond de la vallée était réalisée à l'aide des photographies aériennes. Les résultats obtenus sont encourageants (les concordances entre ceux obtenus par la modélisation et ceux relevés sur le terrain ont atteint approximativement $70 \%$ ) et peuvent être cartographiés au 1:50'000, à une échelle pratique.

PD Dr. Markus Egli, Geographisches Institut, Universität Zürich, Winterthurerstrasse 190, CH-8057 Zürich, Schweiz.

e-mail:megli@geo.unizh.ch

Dipl.-Geogr. Michael Margreth, Geographisches Institut, Universität Zürich, Winterthurerstrasse 190, CH-8057 Zürich, Schweiz.

e-mail: michaelmargreth@yahoo.com

Dr. Urs Vökt, SOILCOM GmbH, Hochmatt 26, CH-3053 Münchenbuchsee, Schweiz.

e-mail:info@soilcom.ch

Prof. Dr. Peter Fitze, Geographisches Institut, Universität Zürich, Winterthurerstrasse 190, CH-8057 Zürich, Schweiz.

e-mail: fitze@geo.unizh.ch

Dipl.-Ing.Agr.ETH Gianfranco Tognina,Amt für Umwelt und Natur, Gürtelstrasse 89, CH-7000 Chur, Schweiz.

e-mail: Gianfranco.Tognina@anu.gr.ch

Dr. Felix Keller, Academia Engiadina, Quadratscha

18, CH-7503 Samedan, Schweiz.

e-mail: felix.keller@academia-engiadina.ch

\section{Manuskripteingang/received/manuscrit entré le} 24.5.2004

Annahme zum Druck/accepted for publication/accepté pour l'impression: 13.6.2005 